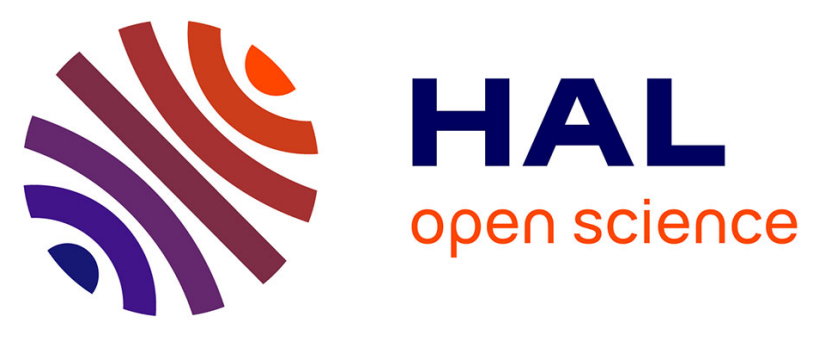

\title{
Prediction of the Biomechanical Effects of Compression Therapy by Finite Element Modeling and Ultrasound Elastography
}

Fanny Frauziols, Jérôme Molimard, Laurent Navarro, Pierre Badel, Magalie Viallon, Rodolphe Testa, Stéphane Avril

\section{To cite this version:}

Fanny Frauziols, Jérôme Molimard, Laurent Navarro, Pierre Badel, Magalie Viallon, et al.. Prediction of the Biomechanical Effects of Compression Therapy by Finite Element Modeling and Ultrasound Elastography. IEEE Transactions on Biomedical Engineering, 2015, 62 (4), pp.1011 - 1019. 10.1109/TBME.2014.2378553 . hal-01139174

\section{HAL Id: hal-01139174 \\ https://hal.science/hal-01139174}

Submitted on 3 Apr 2015

HAL is a multi-disciplinary open access archive for the deposit and dissemination of scientific research documents, whether they are published or not. The documents may come from teaching and research institutions in France or abroad, or from public or private research centers.
L'archive ouverte pluridisciplinaire HAL, est destinée au dépôt et à la diffusion de documents scientifiques de niveau recherche, publiés ou non, émanant des établissements d'enseignement et de recherche français ou étrangers, des laboratoires publics ou privés. 


\title{
Prediction of the Biomechanical Effects of Compression Therapy by Finite Element Modeling and Ultrasound Elastography
}

\author{
Fanny Frauziols, Jérôme Molimard, Laurent Navarro, Pierre Badel, Magalie Viallon, Rodolphe Testa, \\ and Stéphane Avril.
}

\begin{abstract}
Goal: In the present study, the biomechanical response of soft tissues from the fascia cruris to the skin is studied in the human leg under elastic compression. Methods: The distribution of elastic moduli in these tissues is measured for a volunteer at inactive and active muscle states using transient ultrasound elastography (TUSE). After registering the elasticity maps against magnetic resonance imaging (MRI) scans of the same volunteer, patient-specific finite element (FE) models are developed for the leg cross-section at inactive and active muscle states. Elastic properties obtained with TUSE are assigned at each Gauss point of the models. The response to $20 \mathrm{mmHg}$ elastic compression is eventually predicted with the models. Results: Results show significantly higher elastic moduli in the fascia cruris tissue and also a significant increase of elastic moduli at active muscle state. Conclusion: This seems to have a marginal impact on pressure maps in the soft tissues of the leg predicted by the FE models. There is still an effect on the reduction of vein diameter induced by elastic compression, which is decreased at active muscle state. Significance: The discussion of the paper highlights the benefits of using elastography to reconstruct patient-specific FE models of soft tissues.
\end{abstract}

Index Terms-Medical Compression Stocking, Multi-modality, Soft tissues, Supersonic Shear Imaging.

\section{INTRODUCTION}

\section{A. Medical condition: causes and treatment}

D IFFERENT draining mechanisms prevent blood from stagnating in the legs of healthy subjects, such as the effect of muscular contraction, the activity of the sympathetic nervous system or the venous valve system [1]. In case of default in one of these mechanisms, chronic venous insufficiency (CVI) may appear. Symptoms of CVI range from the feelings of "heavy leg" to ulcers. CVI affects about thirty percent of the French population [2]. Elastic compression of the limb using medical compression stockings (MCS) is a widely used treatment since it is efficient for almost all forms of CVI and can be employed at all stages of the disease [3].

Manuscript submitted September 4, 2014. The authors are grateful to the C.N.R.S. (French Scientific National Research Center) for funding the Ph.D. grant of F.F and IFRESIS for its financial support.

Fanny Frauziols, Jérôme Molimard, Laurent Navarro, Pierre Badel and Stéphane Avril are with the Ecole Nationale Supérieure des Mines, CIS-EMSE, CNRS:UMR5307, LGF, Saint-Etienne, France (e-mail: frauziols@emse.fr).

Magalie Viallon is with the Université de Lyon, CREATIS, UMR CNRS 5220 and INSERM U1044, Lyon, France and with the Université Jean Monnet, Service de Radiologie Hôpital Nord, Saint-Etienne, France.

Rodolphe Testa is with the Université de Lyon, Laboratoire de Physiologie de l'Exercice, EA4338, Saint-Etienne, France.

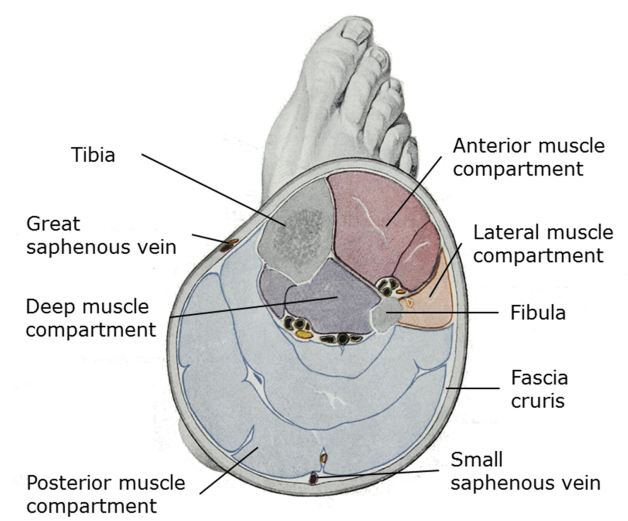

Fig. 1. Anatomy of the human leg - Cross-section of the human right leg. The fascia cruris makes a separation between the superficial tissues, composed by the adipose tissue, the skin and the superficial veins, and the deep tissues, composed by the muscular compartments and the deep veins. Adapted from [4].

\section{B. Motivations for looking at superficial tissues}

The benefits of MCS are not to be proven, but still, for some patients, the therapeutic goal is not reached. It has been shown that the ability of the treatment to achieve the desired medical goal is closely related to the question of transmission of pressure through the soft tissues [5], as a result of which biomechanical models have been developed [6], [7]. These models investigated the transmission of pressure applied with compression garments [7]-[10]. Early studies focused on finding material properties to input in the numerical models [5], [11], [12]. The pressure was found to be heterogeneous inside the leg and with a great inter-subject variability. This gives a first statement on the subject-specific efficacy shown in the literature. Then, studies started to consider the deformation of veins in these models [8], [9], [13]. Recent studies [14] have shown that the biomechanical action and the benefits of elastic compression are different for deep veins and for superficial veins (Fig. 1). Indeed, at active muscle state, the intramuscular pressure in the tissues surrounding deep veins varies by range ten times larger than the pressure applied by MCS. The direct impact of elastic compression on the caliber of deep veins is then relatively limited, especially in the standing position [14]-[16]. The situation is different for superficial veins. When the transmural pressure increases in these veins, their dilatation is only limited by their elastic 
properties and the elastic properties of the surrounding tissues.

\section{Problem definition}

The focus should be on the superficial veins network of the leg when looking at CVI treated by MCS. Also, to account for the inter-subject variability, the mechanical properties of each subject should be used. To that extent, this paper answers the two following questions:

- How do the elastic properties of soft tissues vary from the skin surface to muscle compartments, including the fascia cruris (Fig. 1)?

- Does muscular activation have an impact on the elastic properties of superficial soft tissues?

\section{Proposed solution by the use of a new technology}

Elastography is a non-invasive way of measuring in vivo mechanical properties by inducing a static compression or transient vibrations in soft tissues [17]. A new ultrasound based technology called Supersonic Shear Imaging (SSI) was introduced in 2004 [18], for real-time soft tissue elasticity mapping.

This transient elastography technique is used here for the first time to identify the material property distribution of the soft tissues from the fascia cruris to the skin in the human leg. After registering the obtained elasticity maps against magnetic resonance imaging (MRI) scans, patient-specific finite element (FE) models are developed for entire leg cross-sections at inactive and active muscle states. The response to $20 \mathrm{mmHg}$ elastic compression is eventually predicted with these models.

\section{E. General outlines of the paper}

The material and methods section of this paper give a thorough explanation of the methodology for registering both imaging modalities, and for the implementation of the FE simulations. Then, in the result section, the distributions of the elastic moduli inside the leg at active and inactive muscle states are shown and the responses of the leg under 20 $\mathrm{mmHg}$ of elastic compression for different states of the leg are presented. Finally, these results and the limitations of this study are explained in the discussion section.

\section{Materials And Methods}

\section{A. Data acquisition and processing}

A 22 year old healthy female volunteer underwent a MRI scan (Fig. 2) and SSI ultrasound elastography, following informed consent.

Before acquiring ultrasound data, the subject was asked to perfom an isometric maximum voluntary contraction (MVC), and the response of a torque sensor, attached to the foot, was reported. $10 \%$ of this MVC was taken as baseline for contraction, to ensure good repeatability. An in-house program coded in LabVIEW ${ }^{\circledR}$ (National Instruments, Austin, TX, USA) was used to display the forces and give the volunteer visual feedback to ensure that the desired force was maintained when acquiring ultrasound data.

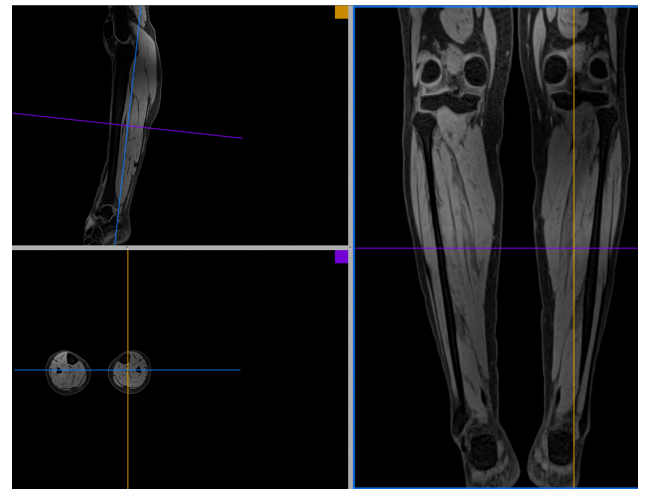

Fig. 2. MRI - Fat-saturated multi-planar of the whole legs of the subject obtained from a high resolution 3D fast dual-echo sequence (Dixon [19], [20]). Imaging parameters were as follows: repetition time (TR) $6.94 \mathrm{~ms} 2.45$ $\mathrm{ms} 3.675 \mathrm{~ms} 5.69 \mathrm{~ms}$, echo time (TE) $2.45 \mathrm{~ms} / 3.675 \mathrm{~ms}$, flip angle 9 degrees, field of view (FOV) $330 \mathrm{~mm}$ x $330 \mathrm{~mm}$, acquisition matrix 320 x 320, number of partitions 176 , bandwidths $500 / 820 \mathrm{~Hz} /$ pixel, parallel acquisition acceleration factor 2 (grappa technique). The scan time per step was $3 \mathrm{~min}$ $24 \mathrm{sec}$. Subjects were imaged in supine feet first position with the arms along the body. Care was taken to avoid any compression of the leg muscle on the MR table when positioning the subject.

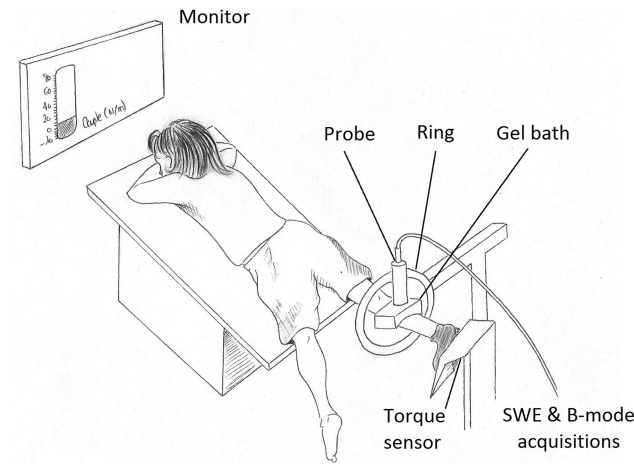

Fig. 3. Experimental Setup - The probe is mounted on a ring, which allows collecting images around the leg. The foot is fixed through a shoe to a torque sensor. A feedback of the torque is given to the subject with the monitor. Data, including B-mode scans and SWE maps are acquired with Aixplorer ${ }^{\circledR}$ (SuperSonic Imagine, Aix-en-Provence, France).

An ultrasound elastographic device (Aixplorer ${ }^{\circledR}$; SuperSonic Imagine, Aix-en-Provence, France) was used to acquire the distribution of elastic moduli in the soft tissues of the leg. In the rest of the article, a distinction will be made between B-mode scans and Shear Wave Elasticity (SWE) maps. The B-mode scans (conventional echography) account for the anatomy of the leg, corresponding to the echogenicity of the medium displayed in gray scale. The SWE maps display the elastic moduli calculated from the velocity fields of shear waves inside the medium, displayed in a false color scale.

An experimental setup was designed so as to hold the ultrasound probe at different positions all around the leg (Fig. 3). A special attention was paid to ensure that the plane of acquisition remains perpendicular to the tibial axis. To ensure minimal movement of the subject, her right shoe was attached to the plate. To ensure that the probe applies minimal compression to the tissues, a small gel bath was designed to place the probe at approximately half a centimeter from the skin. The probe is doing a $360^{\circ}$ rotation around the leg. 
At each position around the leg, SWE and B-mode scans were simultaneously acquired, using a $4-15 \mathrm{MHz}$ probe with 256 piezoelectric elements (SuperLinear ${ }^{T M}$ SL15-4), first at inactive muscle state, second at active muscle state corresponding to $10 \%$ MVC. This required several consecutive acquisitions at different angular positions denoted $\beta_{i}, 1 \leq i \leq N(N=37)$. Adjacent pairs of B-mode scans, say $I_{i}$ and $I_{i-1}$ acquired at angles $\beta_{i}$ and $\beta_{i-1}$, were then stitched together using an inhouse program coded in Matlab ${ }^{\circledR}$.

After picking up a minimum of four anatomical landmarks (veins, crossing of aponeuroses) in each pair of adjacent images, the rigid transformation $F_{i}^{0}$ relating the coordinate systems of both adjacent images was calculated by leastsquares minimization. Then, a cross-correlation factor was estimated across the overlapping area and this cross-correlation factor was optimized by updating the rigid transformation through the Nelder-Mead algorithm [21], yielding finally the rigid transformation $F_{i}$. Repeating the procedure throughout $2 \leq i \leq N$, all pairs of adjacent images were consecutively stitched together. Aligning the coordinate system of $I_{1}$ with the global coordinate system, the rigid transformation $G_{i}=F_{2} \times \ldots \times F_{i}$ was applied to each image $I_{i}$ in order to align it within the global coordinate system.

However, additional constraints had to be considered to ensure an optimal cross-correlation between $I_{1}$ and $G_{N}\left(I_{N}\right)$. Indeed, because the subject could move during the test, the images collected might have come from different transversal planes of the leg. Also, echography has a distortion coming from the width of the beam. Additionally, the stitching process was not fully automatic (points picked by user), thus, user dependent error could occur. To correct these errors, several options were considered (For details, see appendix A).

- Option 1. Find a rigid transformation $F$ such that by applying $F \times F_{i}$ instead of $F_{i}$ to each $I_{i}, G_{N}\left(I_{N}\right)=$ $F \times F_{2} \times \ldots \times F \times F_{i} \times \ldots \times F \times F_{N}\left(I_{N}\right)$ maximizes the cross-correlation between $I_{1}$ and $I_{N}$.

- Option 2. Model the obtained unclosed contour of the leg as a curved beam and deform it until closure. This yields a set of transformations $F_{i}^{b}$ for each image $I_{i}$ ensuring that $F_{2}^{b} \times F_{2} \times \ldots \times F_{i}^{b} \times F_{i} \times \ldots \times F_{N}^{b} \times F_{N}=\iota$ with $\iota$ the identical transformation.

- Option 3. Minimize a global factor:

$$
\bar{d}=d\left(I_{1}, G_{N}\left(I_{N}\right)\right)+\sum_{i=1}^{N-1} d\left(G_{i}\left(I_{i}\right), G_{i+1}\left(I_{i+1}\right)\right)
$$

With $d\left(I_{a}, I_{b}\right)$ the objective function to be minimized representing the distance between anatomical landmarks supposed to be on top of each other after the transformation of successive images. Using the Nelder-Mead algorithm, $\bar{d}$ was minimized by updating the parameters of the $G_{i}$ rigid transformations, for all the transformations simultaneously.

For each angular position denoted $\beta_{i}, 1 \leq i \leq N,(N=37)$, four SWE maps were acquired and averaged, yielding an elasticity image denoted $J_{i}$. Then, each image was aligned within the global coordinate system by applying the rigid

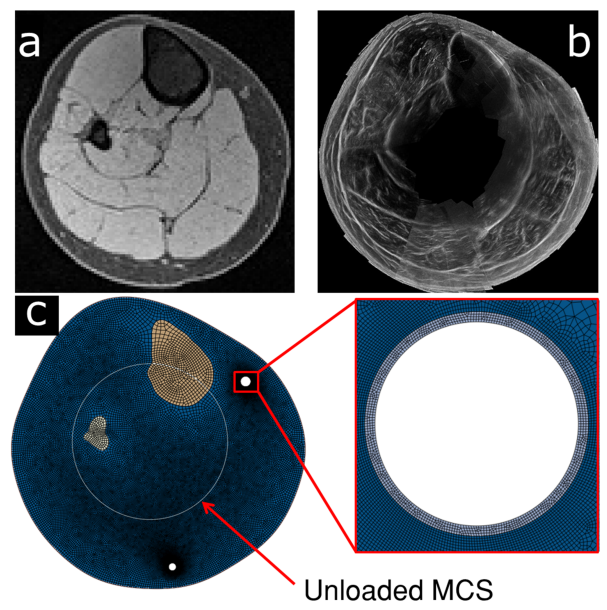

Fig. 4. Finite element mesh of the 2D patient-specific geometry (c) reconstructed from the segmentation of the transversal cross-section of the subject right leg acquired by MRI (a) for the bone and veins and from the ultrasound assembly (b) for the skin. It consists of continuum plane strain elements, except for skin and MCS, composed of truss elements. A relatively finer discretization is used in the vicinity of the great saphenous and small saphenous vein walls (c)

transformation: $G_{i}\left(J_{i}\right)\left(G_{1}=\iota\right)$, permitting a stitched reconstruction of the whole distribution of elastic moduli.

\section{B. FE model implementation}

The MRI leg cross-section and the ultrasound reconstructions (B-mode and SWE) were registered using the bones edges (tibia and fibula) visible in both imaging modalities. From the skin segmentation of the B-mode reconstruction, the global geometry of the leg was defined. The contours and positions of the bones as well as the great and small saphenous veins [22] was added from the segmentation of the MRI leg cross-section. This geometry was used to create a 2D FE mesh, made of 4-node plane strain elements, using the meshing tools available in Abaqus ${ }^{\circledR}$ (Fig. 4). Soft tissues were represented with a Neo-Hookean hyper-elastic constitutive model. Their strain energy per unit of reference volume was defined such as [23]

$$
U=C_{10}\left(\bar{I}_{1}-3\right)+\frac{1}{D_{1}}\left(J^{e l}-1\right)^{2}
$$

With $\bar{I}_{1}$ the first deviatoric strain invariant and $C_{10}$ and $D_{1}$ the material parameters reported in Table I. For each Gauss point from the fascia cruris to the skin, SWE data were available and the following material property was assigned: $C_{10}=\frac{E}{6}$, where $E$ was the locally measured elastic modulus using SWE. Deeper than the fascia cruris a value of $C_{10}=3 \mathrm{kPa}$ was assigned [6] for the model at inactive muscle state. Regarding the model at $10 \%$ MVC, a parametric study was performed on the elastic moduli input deeper than the fascia cruris to evaluate the influence of likely evolving material properties in contracted muscles.

The MCS was defined as a circle of radius equal to the radius of the sock at inactive muscle state. A class II MCS, corresponding to $15-20 \mathrm{mmHg}$ external compression was used. Material properties are reported in Table I. 
TABLE I

MATERIAL PROPERTIES

\begin{tabular}{|c|c|c|c|}
\hline Material & Model & Parameters & Source \\
\hline $\begin{array}{c}\text { Soft tissue inside } \\
\text { fascia cruris }\end{array}$ & $\begin{array}{c}\text { Hyper-elastic } \\
\text { Neo-Hookean }\end{array}$ & $\begin{array}{c}C_{10}=0.005 \mathrm{MPa} \\
D_{1}=0.14 \mathrm{MPa}\end{array}$ & {$[6]$} \\
\hline Soft tissue on and & Hyper-elastic & $\begin{array}{c}C_{10}=\frac{E}{6} \mathrm{MPa} \\
D_{1}=0.14 \mathrm{MPa}\end{array}$ & SSI \\
eutside fascia cruris & Neo-Hookean & $\begin{array}{c}D_{10}=0.1 \mathrm{MPa} \\
D_{1}=0.14 \mathrm{MPa}\end{array}$ & {$[25]$} \\
\hline Skin & $\begin{array}{c}\text { Hyper-elastic } \\
\text { Neo-Hookean }\end{array}$ & $\begin{array}{c}C_{10} \\
\nu=0.49\end{array}$ & {$[7]$} \\
\hline Veins & Linear elastic & $\begin{array}{c}E=0.4 \mathrm{MPa} \\
\nu=0.49\end{array}$ & {$[6]$} \\
\hline MCS & Linear elastic & $\begin{array}{c}E=0.4 \\
\nu\end{array}$ \\
\hline \multicolumn{2}{|c}{}
\end{tabular}

The vein wall was modeled using five elements through the whole $1 \mathrm{~mm}$ thickness. The material properties were linearized following Rohan et al [7].

The simulation entailed two steps. At first, the radius of the MCS was increased until the whole leg fitted in it. Then, this kinematic condition was removed and the contact between skin and MCS was activated. In order to match the experimental conditions where the subject lied supine, a blood pressure of $15 \mathrm{mmHg}$ was applied on the inner surface of the vein walls. Both bones were fixed in the model.

The interaction between the skin and the sock was enforced using a Coulomb frictional behavior with a skin-to-textile friction coefficient fixed at 0.3 as reported in literature [24]. From the Abaqus ${ }^{\circledR}$ library for contact algorithms, the penalty formulation was used in the tangential and normal directions. In the latter, an exponential contact pressure/over-closure relationship was defined.

Two sets of simulations were computed:

- The biomechanical response of the leg to external compression at inactive and active (corresponding to $10 \%$ MVC) muscle states.

- A parametric study to evaluate the influence of the constitutive parameters of the deep soft tissues on the response of superficial soft tissues to elastic compression.

\section{RESULTS}

\section{A. B-mode assembly}

A stitched reconstruction of the $37 \mathrm{~B}$-mode scans without constraining the contour to be closed is shown in Fig. 5. A significant gap remains, which justifies the use of an additional constraint for the stitching approach.

The results obtained with the different stitching approaches providing a closed contour are shown in Fig. 6. The sum of the differences of grayscale values in the overlapping areas were derived for the three options. Their values are 30.2 for option 1, 29.6 for option 2 and 29.4 for option 3. Option 3 is the approach which provides an optimal match between all pairs of images (Fig. 6 - c). The rigid transformations of option 3 are the ones used further to reconstruct the stitched elasticity maps for the leg at inactive and active muscle states (Fig. 7). Stitching errors were finally quantified through a criterion defined as the average distance between 10 anatomical landmarks in each pair of B-mode scans. Average errors for the entire

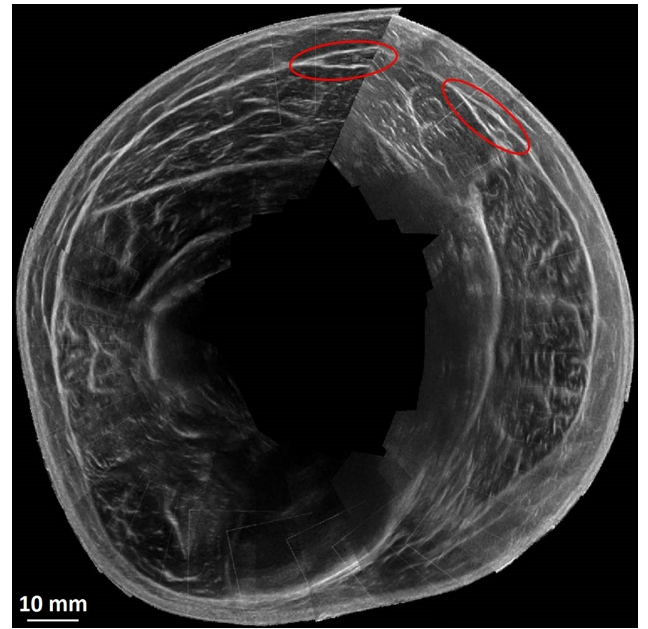

Fig. 5. Initial assembly of B-mode scans - The reconstruction is not closed (red circles).

stitched reconstruction are respectively $0.67 \mathrm{~mm}$ and $0.83 \mathrm{~mm}$ at inactive and active muscle states.

The maps of elastic moduli reconstructed from SWE measurements are showed in Fig. 7 at inactive state and active muscle state corresponding to $10 \%$ MVC. They both are heterogeneous, with overall higher values at the surface (skin) and at the location of fascia cruris.

Elastic moduli (Fig. 7 - b) are globally higher at active muscle state corresponding to $10 \%$ MVC than at inactive muscle state (Fig. 7 - a). A mean value of $12.4 \mathrm{kPa}$ was measured for the elastic moduli (from skin to fascia cruris) at inactive muscle state and $17.2 \mathrm{kPa}$ at active muscle state corresponding to $10 \%$ MVC.

\section{B. Elastic compression of the leg}

The first studied case is for the muscles at inactive muscle state (Fig. 8). Hydrostatic pressure was chosen for the analysis because it does not depend on the coordinate system and can be used to predict local fluid flows.

The second studied case is for the muscles at 10\% MVC. To the best of our knowledge, the literature does not report global $C_{10}$ value for $10 \%$ MVC inside the leg. To analyze the impact of the input value below the fascia cruris in our contracted model, a parametric study increasing this value up to 20 times the value inputted for the model at inactive muscle state was performed. Increasing the elastic moduli of the muscular compartments affects the hydrostatic pressure distributions derived from these simulations by accentuating the tension and compression found between the skin and the fascia cruris, until this inner $C_{10}$ overpasses a threshold value of $90 \mathrm{kPa}$. Then, the effect remains stable (Fig. 9).

For all cases, the obtained maps (Fig. 8 and 9) show heterogeneous distributions. The maximal pressure is located where the curvature radius is minimal (near the tibia) and conversely, minimal pressures are found on flat regions of the leg. An important effect of elastic compression is the impact on the area of the superficial veins. To study this effect, the FE model incorporated two major veins of the leg. For both veins (small 


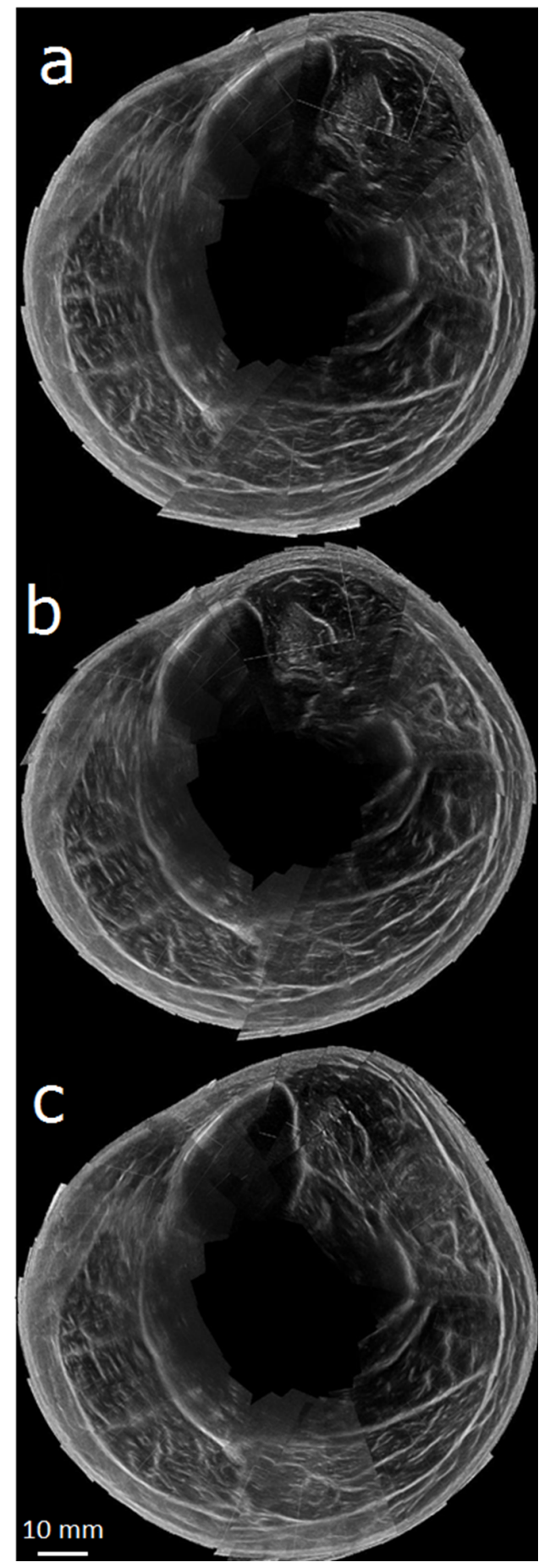

Fig. 6. Closing contour methods comparison : a) option 1 b) option 2 c) option 3 optimization.

and great saphenous veins), the effect of elastic compression is a slight decrease of the vein cross-sectional area (Tables II and III). The decrease is significantly larger for the great saphenous vein than for the small saphenous vein, due to the larger curvature at the location of the great saphenous vein and due to the stiffer properties around the small saphenous vein. Also, the decrease in cross-sectional area diminishes as the elastic modulus increases inside the fascia cruris (Table III).

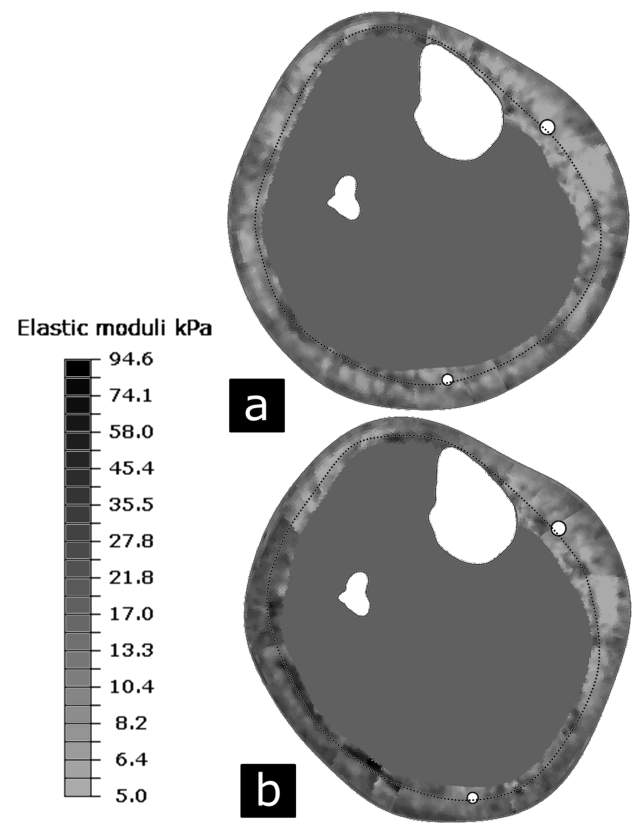

Fig. 7. Elastic moduli distribution in $\mathrm{kPa}$ at inactive muscle state (a) and at active muscle state corresponding to $10 \%$ MVC (b). The position of the fascia cruris is marked as dotted lines.

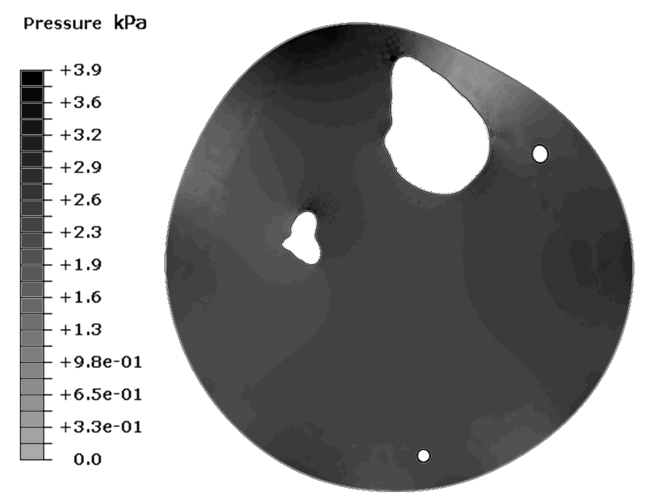

Fig. 8. Hydrostatic pressure distribution in $\mathrm{kPa}$ for the leg at inactive muscle state, during $20 \mathrm{mmHg}$ of elastic compression.

TABLE II

Percentage of Contraction (I.E., DeCREASE of the CROSS-SECTIONAL AREA) OF THE GREAT AND SMALL SAPHENOUS VEINS UNDER AN ELASTIC COMPRESSION OF 15-20 MMHG FOR THE MODEL AT INACTIVE MUSCLE STATE.

\begin{tabular}{|c|c|}
\hline Vein position & Contraction (\%) \\
\hline Great saphenous & 4.5 \\
\hline Small saphenous & 3.7 \\
\hline
\end{tabular}

\section{Discussion}

\section{A. Stitching methodology}

An important drawback of SWE, as well as conventional echography, is the small size of the field of view. Several stitching of B-mode scans from all around the leg were presented here. The grayscale values of the overlapping area for the three options helped us define the best way to stitch 


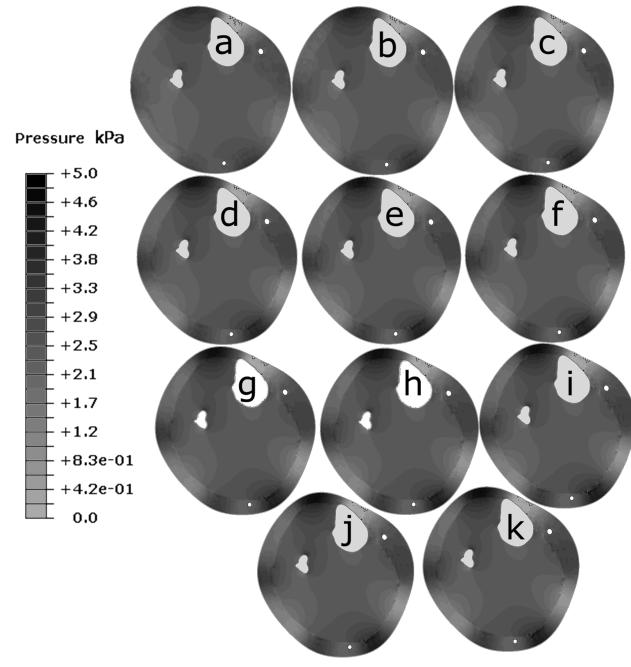

Fig. 9. Parametric study results - Hydrostatic pressure distribution in $\mathrm{kPa}$ for the leg under $20 \mathrm{mmHg}$ of elastic compression. $10 \%$ MVC elastic moduli are used for the fascia cruris up to the skin. Inside the fascia cruris, the value was increased as follow, for cases a to k (in kPa): 18 - 36 - 72 - 108 - 144 $180-216-252-288-324-360$.

TABLE III

PARAMETRIC STUdy. PERCENTAGE OF CONTRACTION (I.E., DECREASE OF THE CROSS-SECTIONAL AREA) OF THE GREAT AND SMALL SAPHENOUS VEINS UNDER AN ELASTIC COMPRESSION OF 15-20 MMHG FOR THE MODEL AT $10 \%$ MVC.

\begin{tabular}{|c|c|c|}
\cline { 2 - 3 } \multicolumn{1}{c|}{} & \multicolumn{2}{c|}{ Contraction (\%) } \\
\cline { 2 - 3 } \multicolumn{1}{c|}{} & Great Saphenous Vein & Small Saphenous Vein \\
\hline $\mathrm{a}$ & 8.1 & 5.7 \\
\hline $\mathrm{b}$ & 8.0 & 5.3 \\
\hline $\mathrm{c}$ & 7.8 & 4.6 \\
\hline $\mathrm{d}$ & 7.8 & 4.2 \\
\hline $\mathrm{e}$ & 7.7 & 3.9 \\
\hline $\mathrm{f}$ & 7.7 & 3.8 \\
\hline $\mathrm{g}$ & 7.7 & 3.6 \\
\hline $\mathrm{h}$ & 7.7 & 3.5 \\
\hline $\mathrm{i}$ & 7.7 & 3.3 \\
\hline $\mathrm{j}$ & 7.6 & 3.4 \\
\hline $\mathrm{k}$ & 7.6 & 3.3 \\
\hline
\end{tabular}

the images. The quantitative spatial error on the stitching gave us a first quality criterion of this methodology. By assembling B-mode scans around the leg, a wide field of view was explored, comparable to the extended field-of-view [26] (EFOV) software, used by clinicians to explore large areas, such as abdomen [27] or musculoskeletal system [28]. However, to the best of our knowledge, extended field-of-view was never performed along with SWE.

Other imaging modalities can overcome this drawback. For example, magnetic resonance elastography has been used to explore elasticity of soft tissue in large anatomical areas such as thighs [17] or livers [29] but due to a low spatial resolution, it is not appropriate to study sub-cutaneous tissues. Moreover, external vibrator is needed to generate low frequency shear waves compared to SSI where the ultrasonic probe remotely generates the shear wave inside the region of interest through pressure of radiation [18] allowing ease of use and minimal discomfort to the subject.

To improve the SWE methodology, a different design of the probe that follows the contour of the leg may be an alternative in the future.

\section{B. Material properties of the subcutaneous tissues}

A mean value of $12.4 \mathrm{kPa}$ was measured for the elastic moduli (from skin to fascia cruris) at inactive muscle state and $17.2 \mathrm{kPa}$ at active muscle state corresponding to $10 \%$ MVC. These are consistent with literature, where values range from 11 to $32 \mathrm{kPa}$ for fat [6], [30], [31]. The two questions to be addressed in the introduction were here answered. The distribution of the elastic moduli in the superficial tissues are heterogeneous and the muscular activation tends to increase the values of the elastic moduli.

The in vivo material properties of subcutaneous tissues have been an intense topic of research for the past 10 years. Measurement of skin elasticity have been performed through traditional tests such as suction [24], [32] or torsion. More recently, to account for anisotropy, micro-robot equipped with force and displacement sensors have been developed and used to demonstrate the strongly anisotropic and viscoelastic behavior of skin [33]. However, these techniques can only give local elasticity measures. Another approach combining finite element analysis, MRI and indentation was able to define more global mechanical parameters for the different layers of the human skin [31] and subcutaneous tissues [30].

All these different methods developed, although promising, cannot address the heterogeneities of mechanical properties found in subcutaneous soft tissues which can be obtained with SWE.

\section{Prediction of the effects of elastic compression}

Rohan et al. [7] performed a computational parametric study and they showed that the biomechanical response of superficial veins to elastic compression is subject to three mechanical factors: the size of the cross-section, the position on the leg contour (local radius of curvature of the leg) and the "type" of fat of the patient. This highlighted the strong patient-specific response of the leg to external compression. By using the elastic moduli measured by SWE, the effect of heterogeneity in material property distribution and the change in elastic moduli of $10 \%$ MVC on pressure maps and veins contraction was determined in this study. This effect remains marginal, though, in the range of moduli measured for the volunteer considered here.

This shows that the distribution of perivenous tissue pressure [34] is mostly determined by the shape and the size of each subject's leg (local radii of curvature) for superficial veins such as the great saphenous veins and the small saphenous veins. The hydrostatic pressure distributions reported here confirm the idea that MCS work towards increasing perivenous tissue pressure on superficial veins consequently reducing the transmural pressure. Trans-mural pressure is also known to be related to the tension of the vein wall according to the Laplace law [35]. Reduction of this tension implies lower alterations in the vein wall associated with various pathologies. 
Several researches [36]-[38] have shown that muscle activation is modified by medical devices similar to MCS. Indeed, skin mechanoreceptors, provide not only exteroceptive by also proprioceptive effects, through Pacinian and Ruffini mechanoreceptors [39]. These are some of the receptors found in the subcutaneous tissue. They sustain essentially static pressure and stretch [40]. In the skin and adipose tissue, inhomogeneous material properties around the locations of the mechanoreceptors may also cause significant differences in the spatial distribution of candidate relevant stimuli of the pressure. Therefore, the inhomogeneous material properties may have a stronger impact on the proprioceptive effects of elastic compression.

\section{Limitations and perspectives}

A question could be raised on the nature of elastic moduli given by SWE in soft tissues. Indeed, one assumption used to retrieve the elastic moduli from shear wave group velocity is that the medium is isotropic and homogenous. It is a fairly common assumption to consider adipose tissue as such [30], [31], [41]. For skeletal muscular tissues, which are made of fibers, it has been previously considered as transversely isotropic [42], [43]. Here, a 2D model in transversal plane is constructed, so muscles can be considered as isotropic. Nevertheless, further investigation would be needed to check the relevance of the elastic moduli given by the Aixplorer ${ }^{\circledR}$ on thin structures such as fascia cruris and skin.

Apart from the assumptions used in SWE, a technical limitation constraining this methodology is not having a good access to deep tissue mechanical properties. This might be overcome by using a probe with a lower frequency that would provide useful information to study the transmission of pressure to deep veins.

A higher level of muscular activation should be investigated to draw strong conclusions on the effects of muscular activation on the transmission of pressure.

\section{CONCLUSION}

In this paper, a new methodology coupling MRI and TUSE data was developed and implemented in a finite element model which is patient-specific in terms of geometry and material properties. This method was used to study the biomechanical response of the leg, under $20 \mathrm{mmHg}$ of elastic compression. The focus was on the material properties of the superficial soft tissues from the skin to the fascia cruris, their impact on the hydrostatic pressure distribution inside the leg and the closure of the great and small saphenous veins.

This opens the way to promising applications for predicting computationally the personalized action of biomechanical devices on soft tissues. 


\section{APPENDIX}

\section{STITCHING ULTRASOUND IMAGES}

Three options were considered to correct the problem of closure of the stitching of all the B-mode scans together.

- Option 1. Find a rigid transformation $F$ such that by applying $F \times F_{i}$ instead of $F_{i}$ to each $I_{i}, G_{N}\left(I_{N}\right)=$ $F \times F_{2} \times \ldots \times F \times F_{i} \times \ldots \times F \times F_{N}\left(I_{N}\right)$ maximizes the cross-correlation between $I_{1}$ and $I_{N}$.

Here, the error was considered to be systematic between all the images. The opening angle was divided by the number of images, and this correcting angle was used to correct the rotational component of the rigid body transformations, then the two other components of the rigid body transformations parameters (the two translations) were computed by maximizing the crosscorrelation between successive images. These three correcting components, the rotation and the two translations are represented by $F$ in the equation.

- Option 2. Model the obtained unclosed contour of the leg as a curved beam and deform it until closure. This yields a set of transformations $F_{i}^{b}$ for each image $I_{i}$ ensuring that $F_{2}^{b} \times F_{2} \times \ldots \times F_{i}^{b} \times F_{i} \times \ldots \times F_{N}^{b} \times F_{N}=\iota$ with $\iota$ the identical transformation.

Here, the error was not considered to be systematic and the idea was to use the finite element method to compute each individual error. Along the fascia cruris, a hundred points were manually picked on the unclosed stitching of the leg (Fig. 10 - a). The first and last points were taken as the same anatomical landmarks. Then, these points were used to define a curved beam on Abaqus ${ }^{\circledR}$. Two boundary conditions were applied. The first boundary condition constrained one end of the line as fixed and the second boundary condition induced a displacement on the other end of the line to make both ends meet. Finally, the finite element simulation provided the displacements and rotations of all points (Fig. $10-$ b). These displacements and rotation, $F_{i}^{b}$, were used to update each previously found rigid body transformations parameters to close the contour of the leg.

- Option 3. Minimize a global factor:

$$
\bar{d}=d\left(I_{1}, G_{N}\left(I_{N}\right)\right)+\sum_{i=1}^{N-1} d\left(G_{i}\left(I_{i}\right), G_{i+1}\left(I_{i+1}\right)\right)
$$

With $d\left(I_{a}, I_{b}\right)$ the objective function to be minimized representing the distance between the anatomical landmarks placed in the global coordinate system which are supposed to be on top of each other. Using the Nelder-Mead algorithm, $\bar{d}$ was minimized by updating the parameters of the $G_{i}$ rigid transformations, for all the transformations simultaneously.

Here, the goal was to find the rigid body transformations parameters globally instead of finding a correcting factor for the previous unclosed stitching. To do so, ten anatomical landmarks were picked on the image $I_{i}$ and the same ten anatomical landmarks were picked on the image $I_{i+1}$. This process was repeated through all the images and circled back to the first one, meaning that ten anatomical
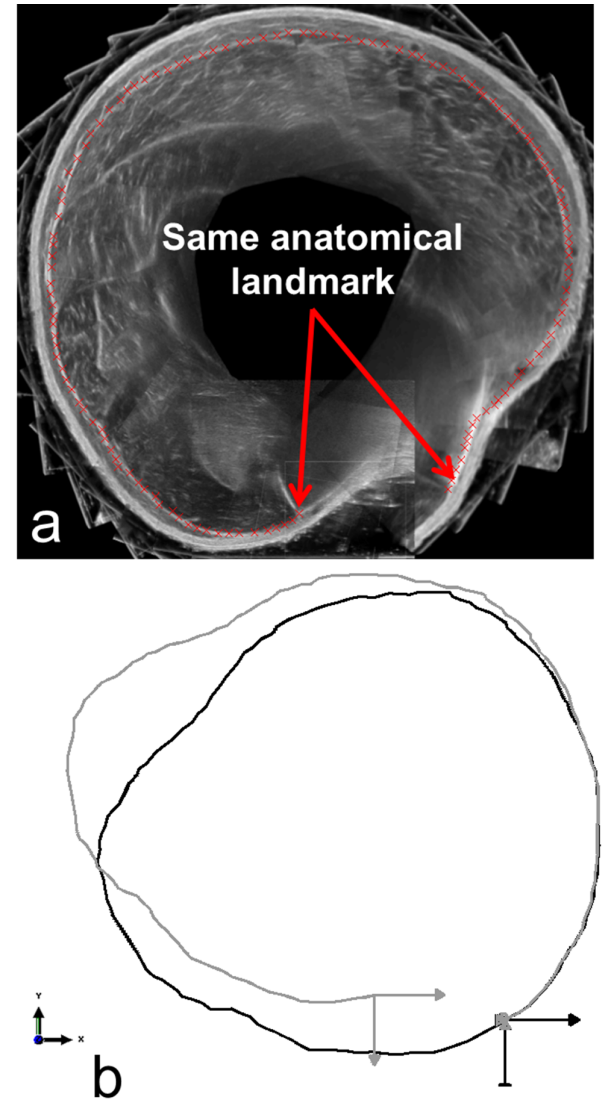

Fig. 10. Schematic overview of the second option of the stitching method. The contour of the aponeurosis is displayed on a stitching of B-mode scans (a). Pre-computation (green) and post-computation (blue) of the closure of the contour by finite element modeling (b).
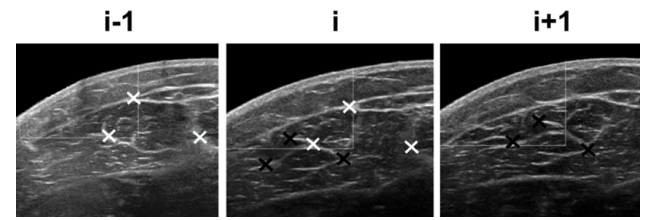

Fig. 11. Schematic overview of the third option of the stitching method. The same anatomical landmarks are picked for each image of all the pairs of images.

landmarks were picked on the image $I_{N}$ and the same ten anatomical landmarks were picked on the image $I_{1}$ (Fig. 11). Then, for each pairs of images, $I_{i}$ and $I_{i+1}$, the rigid body transformation parameters $G_{i}$ were computed and all the points picked were placed in the same global coordinate system. Following this initial estimate of $G_{i}$, an objective function, $\bar{d}$, was defined as the distance of each anatomical landmarks in the global coordinate system which are supposed to be on top of each other. Using the Nelder-Mead algorithm, $\bar{d}$ was minimized by updating the rigid body transformations parameters $G_{i}$. 


\section{ACKNOWLEDGMENT}

The authors want to thank Pr. Rodolphe Le Riche for his support in developing the stitching methodology and the IVTV platform for its experimental support.

\section{REFERENCES}

[1] E. N. Marieb, Anatomie et physiologie humaine, ERPI paris ed. SaintLaurent (Québec): Pearson, 2010.

[2] N. C. Floury, Données sociales 1996 : la société française, INSEE ed., ser. Collection de l'INSEE., Paris, 1996.

[3] H. Partsch, "Chapter 10 - mechanism and effects of compression therapy," in The Vein Book. Burlington: Academic Press, 2007, pp. 103-109.

[4] H. Braus and C. Elze, Anatomie des menschen: ein lehrbuch für studierende und ärzte... J. Springer, 1921, vol. 1.

[5] S. Avril, L. Bouten, L. Dubuis, S. Drapier, and J.-F. Pouget, "Mixed experimental and numerical approach for characterizing the biomechanical response of the human leg under elastic compression," J Biomech Eng, vol. 132, no. 3, p. 031006, Mar. 2010.

[6] L. Dubuis, S. Avril, J. Debayle, and P. Badel, "Identification of the material parameters of soft tissues in the compressed leg," Comput Methods Biomech Biomed Engin, vol. 15, no. 1, pp. 3-11, 2012.

[7] C. P.-Y. Rohan, P. Badel, B. Lun, D. Rastel, and S. Avril, "Biomechanical response of varicose veins to elastic compression: a numerical study," $J$ Biomech, vol. 46, no. 3, pp. 599-603, Feb. 2013.

[8] G. Dai, J. P. Gertler, and R. D. Kamm, "The effects of external compression on venous blood flow and tissue deformation in the lower leg," J Biomech Eng, vol. 121, no. 6, pp. 557-564, Dec. 1999.

[9] A. J. Narracott, G. W. John, R. J. Morris, J. P. Woodcock, D. R. Hose, and P. V. Lawford, "A validated model of calf compression and deep vessel collapse during external cuff inflation," IEEE Trans Biomed Eng, vol. 56, no. 2, pp. 273-280, Feb. 2009.

[10] Y. Wang, I. Pierce, P. Gatehouse, N. Wood, D. Firmin, and X. Y. $\mathrm{Xu}$, "Analysis of flow and wall shear stress in the peroneal veins under external compression based on real-time mr images," Medical engineering \& physics, vol. 34, no. 1, pp. 17-27, 2012.

[11] L. Bouten, "Identification des propriétés mécaniques des tissus constitutifs du mollet pour l'étude mécanique de la contention," Ph.D. dissertation, Ecole Nationale Supérieure des Mines de Saint-Etienne, Mar. 2009.

[12] S. Avril, P. Badel, L. Dubuis, P.-Y. Rohan, J. Debayle, S. Couzan, and J.-F. Pouget, "Patient-specific modeling of leg compression in the treatment of venous deficiency," in Patient-Specific Modeling in Tomorrow's Medicine, ser. Studies in Mechanobiology, Tissue Engineering and Biomaterials, A. Gefen, Ed. Springer Berlin Heidelberg, Jan. 2012, no. 09, pp. 217-238.

[13] Y. Wang, S. Downie, N. Wood, D. Firmin, and X. Y. Xu, "Finite element analysis of the deformation of deep veins in the lower limb under external compression," Med Eng Phys, vol. 35, no. 4, pp. 515-523, Apr. 2013.

[14] P.-Y. Rohan, P. Badel, B. Lun, D. Rastel, and S. Avril, "Prediction of the biomechanical effects of compression therapy on deep veins using finite element modelling," Annals of biomedical engineering, pp. 1-11, 2014.

[15] B. Partsch and H. Partsch, "Calf compression pressure required to achieve venous closure from supine to standing positions," J. Vasc. Surg., vol. 42, no. 4, pp. 734-738, Oct. 2005.

[16] H. Partsch, G. Mosti, and F. Mosti, "Narrowing of leg veins under compression demonstrated by magnetic resonance imaging (mri)." International angiology: a journal of the International Union of Angiology, vol. 29, no. 5, pp. 408-410, 2010.

[17] S. F. Bensamoun, S. I. Ringleb, L. Littrell, Q. Chen, M. Brennan, R. L. Ehman, and K.-N. An, "Determination of thigh muscle stiffness using magnetic resonance elastography," J Magn Reson Imaging, vol. 23, no. 2, pp. 242-247, Feb. 2006.

[18] J. Bercoff, M. Tanter, and M. Fink, "Supersonic shear imaging: a new technique for soft tissue elasticity mapping," IEEE Transactions on Ultrasonics, Ferroelectrics and Frequency Control, vol. 51, no. 4, pp. $396-409$, Apr. 2004.

[19] W. T. Dixon, "Simple proton spectroscopic imaging," Radiology, vol. 153, no. 1, pp. 189-194, Oct. 1984.

[20] J. K. Lee, W. T. Dixon, D. Ling, R. G. Levitt, and W. A. Murphy, Jr, "Fatty infiltration of the liver: demonstration by proton spectroscopic imaging. preliminary observations," Radiology, vol. 153, no. 1, pp. $195-$ 201, Oct. 1984
[21] J. A. Nelder and R. Mead, "A simplex method for function minimization," Computer journal, vol. 7, no. 4, p. 308-313, 1965.

[22] F. Vin, "Varices," EMC - Cardiologie-Angéiologie, vol. 2, no. 1, pp. $1-26,2005$.

[23] K. Hibbit, "Abaqus theory and user manuals version 6.9," USA: ABAQUS Inc, 2009.

[24] L. c. Gerhardt, A. Lenz, N. D. Spencer, T. Münzer, and S. Derler, "Skin-textile friction and skin elasticity in young and aged persons," Skin Research and Technology, vol. 15, no. 3, pp. 288-298, Aug. 2009.

[25] J. T. Iivarinen, R. K. Korhonen, P. Julkunen, and J. S. Jurvelin, "Experimental and computational analysis of soft tissue stiffness in forearm using a manual indentation device," Medical engineering \& physics, vol. 33 , no. 10 , pp. $1245-1253,2011$

[26] L. Weng, A. P. Tirumalai, C. M. Lowery, L. F. Nock, D. E. Gustafson, P. L. Von Behren, and J. H. Kim, "US extended-field-of-view imaging technology," Radiology, vol. 203, no. 3, pp. 877-880, Jun. 1997.

[27] S. H. Kim, B. I. Choi, K. W. Kim, K. H. Lee, and J. K. Han, "Extended field-of-view sonography advantages in abdominal applications," JUM, vol. 22, no. 4, pp. 385-394, Apr. 2003.

[28] E. C. Lin, W. D. Middleton, and S. A. Teefey, "Extended field of view sonography in musculoskeletal imaging." JUM, vol. 18, no. 2, pp. 147152, Feb. 1999.

[29] S. F. Bensamoun, L. Wang, L. Robert, F. Charleux, J.-P. Latrive, and M.-C. Ho Ba Tho, "Measurement of liver stiffness with two imaging techniques: magnetic resonance elastography and ultrasound elastometry," J Magn Reson Imaging, vol. 28, no. 5, pp. 1287-1292, Nov. 2008.

[30] E. Linder-Ganz, N. Shabshin, Y. Itzchak, and A. Gefen, "Assessment of mechanical conditions in sub-dermal tissues during sitting: a combined experimental-MRI and finite element approach," J Biomech, vol. 40, no. 7, pp. 1443-1454, 2007.

[31] H. V. Tran, F. Charleux, M. Rachik, A. Ehrlacher, and M. C. Ho Ba Tho, "In vivo characterization of the mechanical properties of human skin derived from MRI and indentation techniques," Comput Methods Biomech Biomed Engin, vol. 10, no. 6, pp. 401-407, Dec. 2007.

[32] F. Hendriks, D. Brokken, C. Oomens, D. Bader, and F. Baaijens, "The relative contributions of different skin layers to the mechanical behavior of human skin in vivo using suction experiments," Medical Engineering \& Physics, vol. 28, no. 3, pp. 259-266, 2006.

[33] C. Flynn, A. Taberner, and P. Nielsen, "Mechanical characterisation of in vivo human skin using a $3 \mathrm{~d}$ force-sensitive micro-robot and finite element analysis," Biomechanics and Modeling in Mechanobiology, vol. 10, no. 1, pp. 27-38, 2011.

[34] C. Gardon-Mollard and A.-A. Ramelet, La compression médicale. Elsevier Masson, Jul. 2008.

[35] R. J. Gusic, M. Petko, R. Myung, J. William Gaynor, and K. J. Gooch, "Mechanical properties of native and ex vivo remodeled porcine saphenous veins," Journal of biomechanics, vol. 38, no. 9, pp. 17701779, 2005.

[36] D. K. Ramsey, P. F. Wretenberg, M. Lamontagne, and G. Németh, "Electromyographic and biomechanic analysis of anterior cruciate ligament deficiency and functional knee bracing," Clinical Biomechanics, vol. 18, no. 1, pp. 28-34, 2003.

[37] D. Théoret and M. Lamontagne, "Study on three-dimensional kinematics and electromyography of acl deficient knee participants wearing a functional knee brace during running," Knee Surgery, Sports Traumatology, Arthroscopy, vol. 14, no. 6, pp. 555-563, 2006

[38] W. J. Kraemer, S. D. Flanagan, B. A. Comstock, M. S. Fragala, J. E. Earp, C. Dunn-Lewis, J.-Y. Ho, G. A. Thomas, G. Solomon-Hill, Z. R. Penwell et al., "Effects of a whole body compression garment on markers of recovery after a heavy resistance workout in men and women," The Journal of Strength \& Conditioning Research, vol. 24, no. 3, pp. 804-814, 2010 .

[39] R. S. Johansson and A. B. Vallbo, "Tactile sensory coding in the glabrous skin of the human hand," Trends in Neurosciences, vol. 6, pp. 27-32, 1983.

[40] J. H. Martin and T. M. Jessell, "Modality coding in the somatic sensory system," Principles of neural science, vol. 3, pp. 341-352, 1991.

[41] F. S. Azar, D. N. Metaxas, and M. D. Schnall, "Methods for modeling and predicting mechanical deformations of the breast under external perturbations," Med Image Anal, vol. 6, no. 1, pp. 1-27, Mar. 2002.

[42] M. Van Loocke, C. G. Lyons, and C. K. Simms, "A validated model of passive muscle in compression," J Biomech, vol. 39, no. 16, pp. 29993009, 2006.

[43] J. A. Weiss, "A constitutive model and finite element representation for transversely isotropic soft tissues," Ph.D. dissertation, Department of Bioengineering, University of Utah, 1994 